\title{
Grid-Synchronization Stability Improvement of Large Scale Wind Farm During Severe Grid Fault
}

\author{
ShaoKang Ma, Student Member, IEEE, Hua Geng, Senior Member, IEEE, Lu Liu, Geng Yang, Senior \\ Member, IEEE, and Bikash C. Pal, Fellow, IEEE
}

\begin{abstract}
Loss of synchronization between wind farm and power grid during severe grid faults would cause wind farm tripping. In this paper, the mechanism of grid-synchronization is uncovered, described as motion of an autonomous nonlinear differential equation with specific initial states. The revealed mechanism indicates that even though steady state working point exists, improper initial states and poor system dynamic properties could lead to synchronization instability. In order to keep wind farm synchronous with the power grid during severe grid faults, special requirements on system dynamic properties are stated. Moreover, to satisfy all the requirements, a current injecting method is proposed. By adjusting active and reactive output currents of the wind farm, the proposed method could ensure system synchronization stability during severe grid faults. Implementation of the proposed method on PMSG and DFIG based wind farm is illustrated. Simulation results validate the analysis and the control method.
\end{abstract}

Index Terms-Wind farm, grid-synchronization, severe grid fault, nonlinear system, power system stability.

\section{INTRODUCTION}

$\mathbf{E}$ NERGY issues have been discussed worldwide during last few decades. Replacing traditional fossil energy gradually is urgent nowadays. Wind power, as a kind of renewable energy, plays an important role in sustainable generation and will be developed further in the future. Variable speed wind generators are now the most prevalent wind power generators and have been widely adopted in wind farms [1].

The most prevalent control method utilized in variable speed wind generators is vector control. Although alternative control methods have been proposed, vector control is indispensable under certain situations such as ac-fault [2]. Phase-Locked-Loop(PLL), estimating frequency and angle of terminal voltage, is indispensable in vector control [3]. The situation that output frequency, which is determined by the PLL, is identical with the power grid frequency is regarded as grid-synchronization. However, it has been reported that

This work is supported by National High Technology Research and Development Program of China under Grant 2015AA050606, National Key Research and Development Program under Grant 2016YFB0900302, and National Natural Science Foundation of China(NSFC) under Grant U1510208, 61273045 and 51361135705, Technology project of State Grid Corporation of China under Grant NY71-16-052, and EPSRC funded STABLE-NET (EP/L014343/1:).

S. Ma, H. Geng, and G. Yang are with the Department of Automation, Tsinghua University, Beijing 100084, China (e-mail: msk15@mails.tsinghua edu.cn; genghua@tsinghua.edu.cn; yanggeng @tsinghua.edu.cn).

L. Lu is with the School of Automation, Wuhan University of Technology, Wuhan 430070, China (e-mail: liu_717lu@163.com).

Bikash C. Pal is with the Department of Electrical and Electronic Engineering, Imperial College London, London SW7 2BT, U.K. (e-mail: b.pal@imperial.ac.uk).
PLL based vector control has synchronization problems under weak connection [4], [5]. Main difference between strong connection and weak connection is short-circuit ratio(SCR) at the point of common connection(PCC) of wind farm. High line impedance or low grid voltage leads to low SCR, i.e. weakness. Consequently, the terminal voltage of wind farm is more vulnerable under weak connection [6]. Terminal voltage of wind farm is the input of PLL, while the dynamic of PLL determines the injected currents of wind farm, which will in turn affect the terminal voltage [7]. The weakness aggravates the interaction between wind farm and power grid, which significantly influences grid-synchronization. Hence, a lower grid voltage level and a larger grid impedance will result in a less stable situation of grid-synchronization [8].

It is important for variable speed wind generators to have capability of low voltage ride-through(LVRT) when ac-system short-circuit faults occur [9]-[11]. However, during severe short-circuit faults, connection between wind farm and power system is very weak because the low terminal voltage makes SCR at PCC of wind farm very low. Consequently, the synchronization issues between wind farm and power grid would arise during the faults. Hence, in addition to traditional LVRT techniques, special considerations on synchronization stability should be taken. Since the interaction between wind farm and power system under weak connection will cause the grid-synchronization problem, merely improving PLL tracking ability [12]-[15] is insufficient to ensure grid-synchronization. To solve this problem, the interaction should be taken into consideration. PLL and other relative parts, such as current injecting strategy and line impedance, should be analyzed as a whole system. It should be emphasized that gridsynchronization is a general issue associated with grid-tied power electronic equipments [16].

Boroyevich's team has been working on this problem for a long time and good progress has been made in understanding grid-synchronization [8], [17]-[19]. In [8], [19], a simplified large-signal system model is proposed to analyze gridsynchronization of voltage source converters. Based on the simplified model, a synchronization stability criterion is also proposed. In [5], loss of synchronization between full-scale converter based wind farm and power grid is identified under a severe grid fault. Based on the synchronization stability criterion proposed in [19], [5] also derived transfer limitation of wind farm active current and reactive current during severe ac faults. However, the synchronization stability criterion is established under steady state. Since dynamic behavior also affects system stability, the steady state criterion is not adequate, which would 


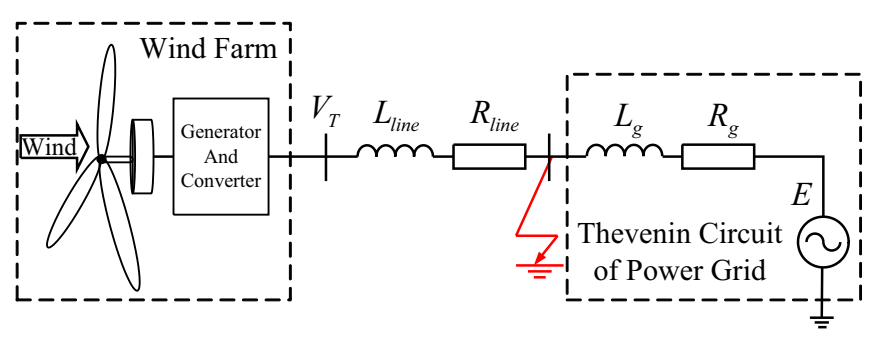

Fig. 1. System configuration.

be discussed further in later section.

As mentioned above, previous works have already pointed out that the coupling between PLL itself and line impedance may destabilize grid-synchronization. However, the dynamic process of synchronization between wind farm and power grid has not been fully investigated and understood. This paper uncovers the mechanism of grid-synchronization, indicating that the dynamic behavior may destabilize the system, even though the system is judged stable based on the steady state synchronization stability criterion. Hence, requirements on system dynamic behavior and additional methods are proposed to enhance grid-synchronization.

\section{SySTEM MODELING}

\section{A. Model of Grid-tied Wind Farm}

The model system is a grid connected wind farm, as shown in Fig.1. The frequency deviation of the power system is omitted in this paper. Hence, the power grid is represented by its Thevenin equivalent circuit, where voltage source $E$ is regarded as ideal with constant voltage and constant frequency. Wind generators in the wind farm are aggregated into one single machine [20], [21]. $L_{\text {line }}$ and $R_{\text {line }}$ represent the inductance and resistance of transmission line from the wind farm to the power grid, respectively.

This paper focuses on the synchronization between wind farm and power grid during fault. According to the grid code, wind generator should inject required reactive current during fault [22]. Generally, bandwidth of the current loops is much higher than the PLL. Hence, the wind generator can be regarded as an ideal controlled current source during fault steady state [5], [8], [23]. Electromagnetic behaviors of transmission line and grid impedance are also omitted.

The system could be represented as Fig.2(a). Resistance in dashed line is utilized to represent short-circuit resistance. Symbols in Fig.2(a) are defined in (1).

$$
\left\{\begin{array}{l}
\delta=\theta_{p l l}-\theta_{g} \\
\theta_{g}=\int_{0}^{t} \omega_{g} d t=\int_{0}^{t} 2 \pi f_{n} d t \\
\theta_{p l l}=\int_{0}^{t} \omega_{p l l} d t=\int_{0}^{t} 2 \pi f_{p l l} d t \\
\bar{I}_{c}^{p}=I_{d}^{p}+j I_{q}^{p}=\left|I_{c}^{p}\right| e^{j \phi_{c}} \\
\bar{E}=E_{d}+j 0=|E|
\end{array}\right.
$$

where $\omega_{g}$ and $f_{n}$ are the electric angular velocity and frequency of power system, respectively. $\omega_{p l l}$ and $f_{p l l}$ are the

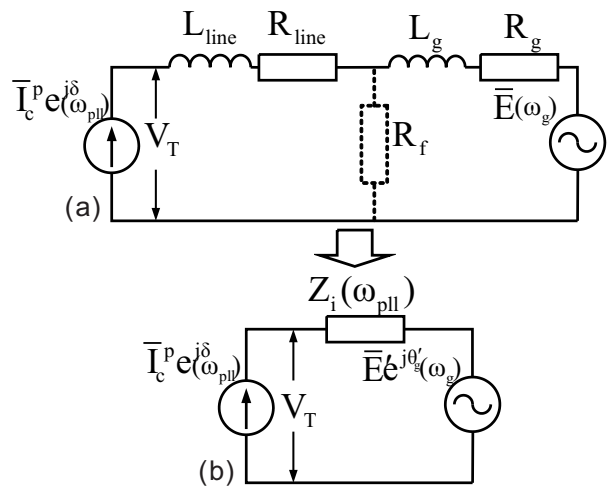

Fig. 2. Simplified representation of the system.

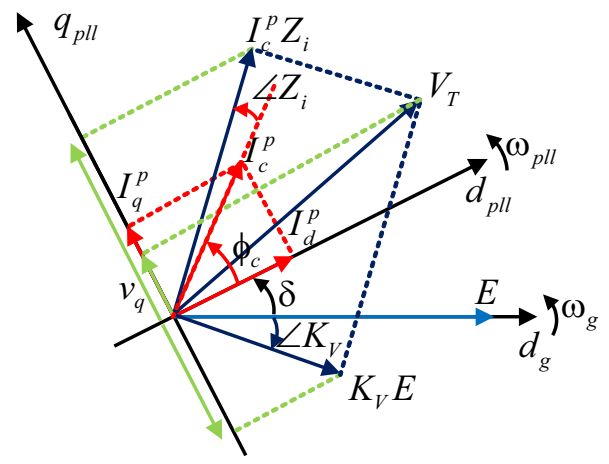

Fig. 3. Relationship between the variables.

electric angular velocity and frequency of PLL, respectively. The initial value of $\theta_{p l l}$ and $\theta_{g}$ are all assumed zero in (1). $\bar{I}_{c}^{p}$ represents the injected currents of wind farm and $\bar{E}$ is the grid voltage. Note that $\bar{I}_{c}^{p}$ is defined on the PLL frame while $\bar{E}$ is defined on the grid voltage frame. The angle difference between the two frame is $\delta$. The two sources with different frequencies contribute to the terminal voltage $V_{T}$ of wind farm. Based on the superposition principle, we can derive

$$
V_{T}=Z_{i}\left(\omega_{p l l}\right)\left|I_{c}^{p}\right| e^{j\left(\phi_{c}+\delta\right)}+K_{v}\left(\omega_{g}\right) E
$$

When operating under normal condition, $R_{f}$ in dashed line is omitted. Thus

$$
\left\{\begin{array}{l}
Z_{i}\left(\omega_{p l l}\right)=\left(R_{\text {line }}+R_{g}\right)+j \omega_{\text {pll }}\left(L_{\text {line }}+L_{g}\right) \\
K_{v}\left(\omega_{g}\right)=1
\end{array}\right.
$$

When the short-circuit fault occurs, $Z_{i}\left(\omega_{p l l}\right)$ and $K_{v}\left(\omega_{g}\right)$ are expressed as

$$
\left\{\begin{array}{l}
Z_{i}\left(\omega_{\text {pll }}\right)=R_{\text {line }}+j \omega_{\text {pll }} L_{\text {line }}+\frac{\left(R_{g}+j \omega_{p l l} L_{g}\right) R_{f}}{R_{f}+R_{g}+j \omega_{p l l} L_{g}} \\
K_{v}\left(\omega_{g}\right)=\frac{R_{f}}{R_{f}+R_{g}+j \omega_{g} L_{g}}
\end{array}\right.
$$

The widely used synchronous reference frame PLL is illustrated in Fig.4. $V_{T}$ is the input of PLL and $\theta_{p l l}$ is the rotation angle of abc to dq transformation. The relationship between 


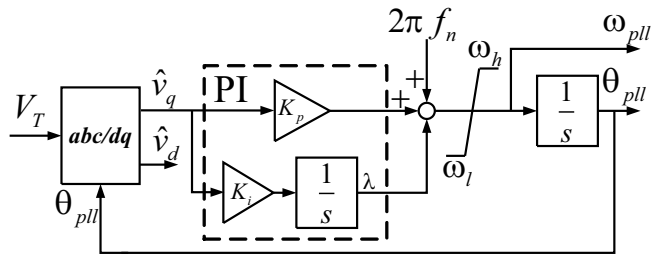

Fig. 4. Block diagram of PLL.

the variables is shown in Fig.3. Assuming that three phase is balanced, $\hat{v}_{q}$ could be derived as (5) [8]

$$
\begin{aligned}
\hat{v}_{q}= & \left|Z_{i}\left(\omega_{p l l}\right) I_{c}^{p}\right| \sin \left(\phi_{c}+\angle Z_{i}\left(\omega_{p l l}\right)\right) \\
& +\left|K_{v}\left(\omega_{g}\right) E\right| \sin \left(\angle K_{v}\left(\omega_{g}\right)-\delta\right)
\end{aligned}
$$

where $\omega_{g}$ is constant. Hence, the first term of right side of (5), which is generated according to PLL angle, can be regarded as dc in PLL frame. We can further simplify expression (5) as

$$
\hat{v}_{q}=a\left(\omega_{p l l}\right)+b \sin \left(\phi_{0}-\delta\right)
$$

where

$$
\left\{\begin{array}{l}
a\left(\omega_{p l l}\right)=\left|Z_{i}\left(\omega_{p l l}\right) I_{c}^{p}\right| \sin \left(\phi_{c}+\angle Z_{i}\left(\omega_{p l l}\right)\right) \\
b=\left|K_{v}\left(\omega_{g}\right) E\right| \\
\phi_{0}=\angle K_{v}\left(\omega_{g}\right)
\end{array}\right.
$$

The system model could be deduced as a second-order nonlinear differential equations, as shown in (8).

$$
\left\{\begin{array}{l}
\frac{d \lambda}{d t}=K_{i} \hat{v}_{q} \\
\frac{d \delta}{d t}=K_{p} \hat{v}_{q}+\lambda \\
\hat{v}_{q}=a\left(\omega_{p l l}\right)+b \sin \left(\phi_{0}-\delta\right)
\end{array}\right.
$$

where $\lambda$ is the value of the integrator of PLL. $K_{p}$ and $K_{i}$ are the parameters of PI regulator of PLL, as shown in Fig.4. Note that the state variable of the second row of (8) is $\delta=\theta_{p l l}-\theta_{g}$ instead of $\theta_{p l l}$. Relationship between angle $\delta$ and PLL input $\hat{v}_{q}$ reflects the interaction between wind farm and power grid.

\section{B. System Weakness During Grid Faults}

In (2), if we set

$$
\left\{\begin{array}{l}
\bar{E}^{\prime}=E_{d}^{\prime}+j 0=\left|E^{\prime}\right|=\left|K_{v}\left(\omega_{g}\right) E\right| \\
\theta_{g}^{\prime}=\angle K_{v}\left(\omega_{g}\right)
\end{array}\right.
$$

then Fig.2(a) is equivalent to Fig.2(b), in which the wind farm connects to another voltage source $\bar{E}^{\prime}$ with grid impedance $Z_{i}\left(\omega_{p l l}\right)$. Based on $\bar{E}^{\prime}$ and $Z_{i}\left(\omega_{p l l}\right)$, we can conveniently calculate short-circuit capacity at PCC of the wind farm.

When the grid fault occurs, the short-circuit capacity is

$$
\begin{aligned}
S_{s c}^{f} & =\frac{\left|E^{\prime}\right|^{2}}{\left|Z_{i}\left(\omega_{g}\right)\right|}=\frac{\left|K_{v}\left(\omega_{g}\right) E\right|^{2}}{\left|Z_{i}\left(\omega_{g}\right)\right|} \\
& =\left|\frac{K_{v}\left(\omega_{g}\right) E^{2}}{\frac{R_{\text {line }}+j \omega_{g} L_{\text {line }}}{K_{v}\left(\omega_{g}\right)}+R_{g}+j \omega_{g} L_{g}}\right|
\end{aligned}
$$

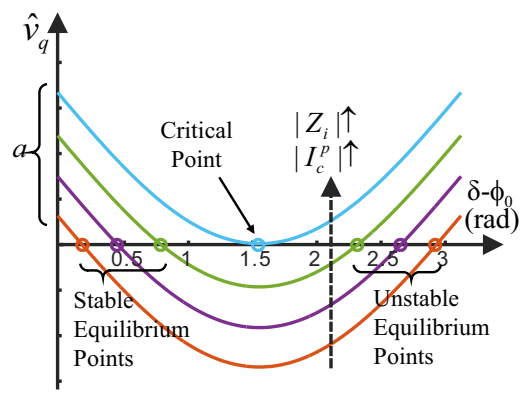

Fig. 5. $\hat{v}_{q}$ curves under different conditions.

while in normal state, the short-circuit capacity is

$$
S_{s c}^{n}=\frac{\left|E^{\prime}\right|^{2}}{\left|Z_{i}\left(\omega_{g}\right)\right|}=\left|\frac{E^{2}}{R_{\text {line }}+j \omega_{g} L_{\text {line }}+R_{g}+j \omega_{g} L_{g}}\right|
$$

Generally, a high grid impedance or a low grid voltage $E$ lead to weak connection. However, from (4), we can obtain that $K_{v}\left(\omega_{g}\right) \ll 1$ when severe fault occurs. Hence, denominator of (10) is much larger than that of (11) and numerator of (10) is much smaller than that of (11). As a result, $S_{s c}^{f}$ is much smaller than $S_{s c}^{n}$. That means even if the prefault power grid is strong(i.e. low grid impedance), the connection could also become weak under severe short-circuit faults where $R_{f}$ is quite small. Consequently, no matter whether the prefault system is strong or not, the synchronization problem between wind farm and power grid should be taken into consideration.

\section{ANALYSIS ON GRID-SYNCHRONIZATION}

Steady state analysis method introduced in this section could be utilized to judge if there are operating points under given conditions. The analysis results are similar to the conclusion obtained via synchronization stability criterion proposed in [8]. Nevertheless, even if steady state working points exist, poor dynamic behavior of system would make these working points unavailable. To clearly understand the essence of this issue, mechanism of grid-synchronization is then revealed, offering a way to evaluate dynamic process of grid-synchronization.

\section{A. Steady State Analysis}

In steady state, PLL frequency $\omega_{p l l}$ equals to power system frequency $\omega_{g}$. From (8) we can obtain that when $\hat{v}_{q}=0$ and $\lambda=0$, the differential equations reach the equilibrium point. Thus, the zero-crossing points of curve $a\left(\omega_{g}\right)+b \sin \left(\phi_{0}-\delta\right)$ could represent the steady state points. Along with the increasing amplitude of line impedance or output currents, amplitude of $\left|a\left(\omega_{g}\right)\right|$, the dc offset of the curve, will increase. When $\left|a\left(\omega_{g}\right)\right|$ is larger than $b, \hat{v}_{q}$ has no zero-crossing point and hence the system is impossible to be stable, as illustrated in Fig.5. On the other hand, if $b>\left|a\left(\omega_{g}\right)\right|$, steady state operating point exists. As shown in Fig.5, $b=\left|a\left(\omega_{g}\right)\right|$ is the critical point from the perspective of steady state. Note that either basic PLL or improved PLLs aim to regulate $\hat{v}_{q}$ to zero [12][15]. Hence, if $\left|a\left(\omega_{g}\right)\right|$ is larger than $b$, the system will lose grid-synchronization no matter how fast and sophisticated the PLL is. 


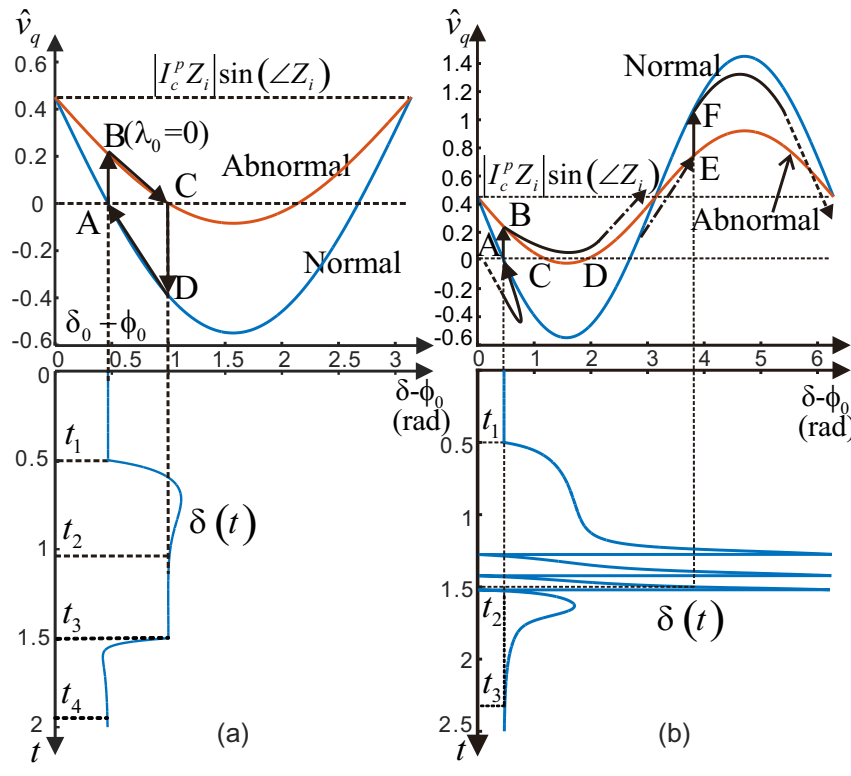

Fig. 6. Illustrations on dynamic process of grid-synchronization.

In Fig.5, each curve has two zero-crossing points. Smallsignal analysis method could be utilized to judge whether a zero-crossing point is stable or not. Assuming $a\left(\omega_{g}\right)$ is constant, the small-signal system matrix and its characteristic polynomial are shown in (12) and (13), respectively.

$$
\begin{gathered}
\mathbf{A}=\left[\begin{array}{ll}
0 & -K_{i} b \cos \left(\delta_{0}-\phi_{0}\right) \\
1 & -K_{p} b \cos \left(\delta_{0}-\phi_{0}\right)
\end{array}\right] \\
\rho_{S}(s)=|s \mathbf{I}-\mathbf{A}| \\
=s^{2}+K_{p} b \cos \left(\delta_{0}-\phi_{0}\right) s+K_{i} b \cos \left(\delta_{0}-\phi_{0}\right)
\end{gathered}
$$

where $\delta_{0}-\phi_{0}$ is the angle of the zero-crossing point. When $\delta_{0}-\phi_{0}<\frac{\pi}{2}$, equation $\rho_{S}(s)=0$ has negative real-part solutions, indicating that the relative zero-crossing point is stable, otherwise unstable. The stable and unstable equilibrium points are shown in Fig.5.

\section{B. Mechanism of Grid-Synchronization}

To study the mechanism of grid-synchronization, extra simulations are demonstrated here. The simulated system is shown in Fig.1. Instead of short-circuit faults, steps of power grid voltage $E$ are applied to mimic abnormal situations in this example for the reason that the obtained curves could have proper scales to be conveniently observed. As a result, expression in (3) rather than (4) should be used at abnormal situations and hence $\phi_{0}=0$ all the time because $K_{v}\left(\omega_{g}\right)=1$ in (3). Moreover, $\bar{I}_{c}^{p}$ is set to 1p.u. active current. Two cases with different power grid voltage variations are simulated in this example, as shown in Fig.6.

The blue sinusoidal curve(i.e. the normal curve) and the red sinusoidal curve(i.e. the abnormal curve) describe the value of $\hat{v}_{q}$ before and after voltage variation, respectively. Fig.6(a) shows that under normal situation, the system works at point A, a steady state working point where $\lambda=0$ and $\delta-\phi_{0}=$ $\delta_{0}-\phi_{0}$. After voltage $E$ drops from 1p.u. to 0.54 p.u. at $t=t_{1}$, the working point steps from A to B immediately. At point
$\mathrm{B}, \lambda=0$ and $\delta$ is the same as point $\mathrm{A}$. However, point $\mathrm{B}$ is not an equilibrium point of the abnormal curve. Thus, $\mathrm{B}$ will move toward $\mathrm{C}$ (i.e. zero-crossing point of the abnormal curve) because $\hat{v}_{q}$ is positive at $\mathrm{B}$, generating increasing trend of $\delta$. After overshooting through point $\mathrm{C}$, system enters the $v_{q}<0$ region and the speed of $\delta$ (i.e. $\omega_{p l l}-\omega_{g}$ ) decreases. $\omega_{p l l}-\omega_{g}$ reaches zero in the $v_{q}<0$ region and subsequently becomes negative. The system will finally settle at point $\mathrm{C}$, a stable point during abnormal stage. The dynamic process can be described by (8) with initial states $\lambda_{0}=0$ and $\delta_{0}-\phi_{0}$. When voltage $E$ is recovered, the working point moves from point $\mathrm{C}$ to point $\mathrm{A}$ in a similar process. In Fig.6(b), voltage $E$ drops from 1p.u. to 0.46p.u. at $t=t_{1}$. Point B on the red curve(i.e. abnormal curve) will move to its zero-crossing point C. Compared to Fig.6(a), the voltage drops deeper and hence the $v_{q}<0$ region is smaller under abnormal situation in Fig.6(b). System reaches point D before $\omega_{p l l}-\omega_{g}$ could decrease to zero. Hence, $\omega_{p l l}-\omega_{g}$ still has a positive value at point $\mathrm{D}$. As a result, $\delta$ finally goes into the positive part of the red curve after crossing point $\mathrm{D}$. The system can never work at point $\mathrm{C}$ stably during abnormal stage in Fig.6(b), which means that synchronization stability of the system cannot be guaranteed. At the moment before the voltage recovers, the system works at point E. After the voltage is recovered, the working point steps from E to F. Finally, the system will work at point A again.

This example offers a basic sight of transient characteristics of grid-synchronization after large disturbance. Note that in this example, $\phi_{0}=0$ all the time. In fact, when a short-circuit fault occurs, expression in (4) should be used. Thus, angle $\phi_{0}$ does not equal to zero during the fault. From the illustrations, we can obtain the following conclusion.

- Theoretically, dynamic process of synchronization between wind farm and power grid after large disturbance could be described as motion of autonomous nonlinear equation (8) with initial states $\lambda_{0}=0$ and $\delta_{0}-\phi_{0} \in$ $[0,2 \pi]$.

- Overshoot may lead to instability even though steady state working point exists, especially when the dc offset is close to $b$ (i.e. the zero-crossing point of $\hat{v}_{q}$ is close to its critical point), as the case in Fig.6(b).

In practice, the initial phase $\delta_{0}-\phi_{0}$ could be any value in $[0,2 \pi]$. The reasons are listed below.

- The value of $\phi_{0}$ in fault state, which is determined by impedance network, is uncertain and different from that in normal state.

- $\delta_{0}$ is undetermined because of detection delay and protection. Take DFIG as a example, $\delta$ would vary randomly when crowbar of DFIG is activated. At the moment rotorside converter is reconnected and output currents are controlled to their commands, which is regarded as the initial moment of $(8), \delta_{0}$ could be any value in $[0,2 \pi]$.

The value of $\lambda_{0}$ is also undetermined at the initial moment because of detection delay and protection. However, since PLL is software based, we can manually reset $\lambda$ to zero whenever needed. Consequently, the assumption that initial state $\lambda_{0}=0$ always holds. 


\section{SYNCHRONIZATION STABILITY IMPROVEMENT During SeVEre Short-Circuit Faults}

\section{A. Synchronization Stability Requirements}

Loss of synchronization is usually regarded as an abnormal state and may lead to failure in power control and wind farm tripping. Hence, to maintain wind farm connected with power grid during grid fault, grid-synchronization should be ensured. Based on the steady state analysis and the mechanism of gridsynchronization mentioned in Section-III, special requirements on grid-synchronization for wind farm during severe shortcircuit faults could be summarized as follow.

- Existence of working point should be ensured so that the wind farm can inject desired reactive current correctly during severe short-circuit faults.

- Dynamic process of grid-synchronization should be ensured stable. That means for every initial states $\lambda_{0}=0$ and $\forall \delta_{0} \in[0,2 \pi]$, the trajectory of system equation (8), representing system behavior during faults, is supposed to converge to its steady state working point.

\section{B. Adaptive Current Injecting Strategy}

Previous works have investigated moderate fault situations where voltages are no lower than 0.1p.u. [9]-[11]. These works show no synchronization problems. However, loss of synchronization is identified at severe fault situation where voltages drop to less than 0.05p.u. [5]. Hence, in this part, severe grid fault situations are considered. An adaptive current injecting strategy is proposed to ensure synchronization stability when voltage drops to a very low value(e.g. below 0.1 p.u.). The proposed method complies with all the special requirements.

The severe short-circuit fault could be represented by a quite small resistance $R_{f}$, as illustrated in Fig.2(a). Since $R_{f}$ is much smaller than line impedance and grid impedance at severe fault situations, it can be deduced from (4) that

$$
Z_{i}\left(\omega_{\text {pll }}\right) \approx R_{\text {line }}+j \omega_{\text {pll }} L_{\text {line }}
$$

and

$$
\angle K_{v}\left(\omega_{g}\right) \approx-\arctan \left(\frac{\omega_{g} L_{g}}{R_{g}}\right)
$$

According to the PLL frequency, if we set output current commands of the wind farm adaptively, as shown in (16)

$$
\left\{\begin{array}{l}
\frac{I_{q}^{p}}{I_{d}^{p}}=-\tan \angle Z_{i}\left(\omega_{\text {pll }}\right)=-\frac{\omega_{\text {pll }} L_{\text {line }}}{R_{\text {line }}} \\
\sqrt{I_{d}^{p^{2}}+I_{q}^{p^{2}}} \leq 1 \text { p.u. }
\end{array}\right.
$$

then

$$
\phi_{c}+\angle Z_{i}\left(\omega_{p l l}\right)=0
$$

which means

$$
a\left(\omega_{p l l}\right)=0
$$

Then (8) could be rewritten as

$$
\left\{\begin{array}{l}
\frac{d \lambda}{d t}=-K_{i} b \sin (\sigma) \\
\frac{d \sigma}{d t}=-K_{p} b \sin (\sigma)+\lambda
\end{array}\right.
$$

where $\sigma=\delta-\phi_{0}$.

The DC offset of $\hat{v}_{q}$ curve is zero under this control method. Hence, working point is bound to exist. To evaluate stability of (19), Lyapunov method is used [24], [25]. Consider the following Lyapunov function

$$
V(\lambda, \sigma)=\frac{1}{2} \lambda^{2}+K_{i} b(1-\cos \sigma) \geq 0
$$

Hence,

$$
\begin{aligned}
\dot{V}(\lambda, \sigma) & =\lambda \dot{\lambda}+K_{i} b \sin \sigma \dot{\sigma} \\
& =-K_{i} b \lambda \sin \sigma-K_{i} K_{p} b^{2} \sin ^{2} \sigma+K_{i} b \lambda \sin \sigma \\
& =-K_{i} K_{p} b \sin ^{2} \sigma
\end{aligned}
$$

It is true that $K_{p}>0$ and $K_{i}>0$. Then we can obtain

$$
\dot{V}(\lambda, \sigma) \leq 0
$$

From Lyapunov stability criterion, system (19) is globally stable, indicating that the adaptive current injecting method could help the wind farm to maintain grid-synchronization.

The wind farm injects active power and reactive power to the grid, simultaneously. Since the terminal voltage of wind farm is very low during fault, the captured mechanical power cannot be dissipated via transmission line even the proposed current injection strategy injects active current. Note that utilizing the adaptive current injecting control, the reactive current cannot reach 1p.u. because the active current is supposed to be set non-zero. Consequently, this control method violates the grid code. However, this dilemma could be avoided by applying auxiliary SVG, which could inject extra reactive current to meet the grid code. If total active current and total reactive current injected by the wind farm and the SVG meet the adaptive current injecting strategy, and the total reactive current is no less than 1p.u., both the synchronization stability and the grid code could be guaranteed.

Under extreme situation where $R_{f}$ equals to zero, the value of $b$ is zero according to (7). Theoretically, utilizing the adaptive current injecting method, the value of $a\left(\omega_{p l l}\right)$ can be controlled to zero. Hence, according to Fig.4, the output frequency of wind farm is determined only by the integrator value $\lambda$ of the PLL. If we manually reset $\lambda$ to zero, the output frequency is $f_{n}$. However, the frequency of power grid may slightly drift from $f_{n}$ during fault. For the cases where $R_{f}$ is not zero, the value of $b$ is not zero and the PLL can capture the frequency information of power grid hence output frequency of wind farm can track the power system frequency. However, if $R_{f}$ is zero, wind farm can no more capture the information of power system frequency. Consequently, even though the proposed current injection method can stabilize the system under $R_{f}=0$ situation, the output frequency of wind farm is not exactly the same as power grid.

Moreover, (19) could also utilized to explain why synchronization stability can be always guaranteed under strong power grid. If line impedance is small, the value of $\left|I_{c}^{p} Z_{i}\left(\omega_{p l l}\right)\right|$ is small compared to the amplitude of terminal voltage $E$. Hence, impact of term $a\left(\omega_{p l l}\right)$ is negligible under strong connection. Based on this assumption and former Lyapunov analysis, synchronization stability could be always ensured. 


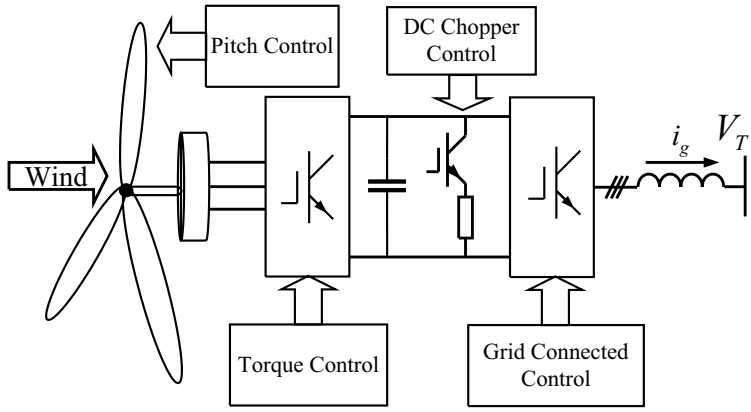

Fig. 7. Structure of PMSG.

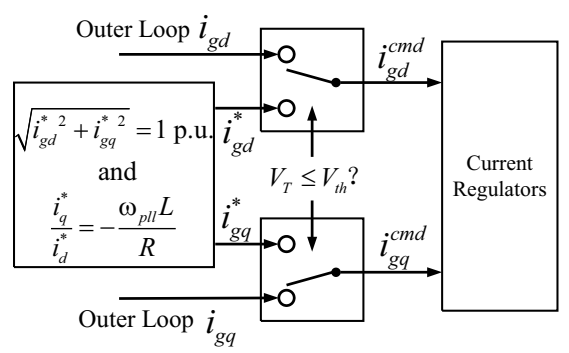

Fig. 8. Current control block diagram of PMSG.

\section{IMPLEMENTATION OF ADAPTIVE CURRENT INJECTING STRATEGY IN PMSG AND DFIG BASED WIND FARM}

\section{A. Implementation of Adaptive Current Injecting Strategy in PMSG Based Wind Farm}

Full-scale converter is applied in PMSG wind farm. The advantage of this structure is the decoupling between gridside converter and wind generator. It is possible for PMSG wind farm to control grid currents independently.

Structure of PMSG is shown as Fig.7. When working under normal state(i.e. no fault occurs), the grid-side converter regulates the dc voltage and the rotor-side converter controls electrical torque of the PMSG according to MPPT curve or upper level command. During short-circuit faults, the dc chopper could release surplus energy in the capacitor in case of over-voltage. Bang-bang control strategy is used to control the dc chopper. Pitch control of wind turbine is utilized to protect the drive train in case of over-speed.

Current control is shown in Fig.8. Under normal state, current commands are obtained by outer control loops. When severe faults occur, the proposed current strategy should be utilized.

\section{B. Implementation of Adaptive Current Injecting Strategy in DFIG Based Wind Farm}

Structure of DFIG is shown in Fig.9. Stator windings of the DFIG are directly connected to the power grid and the wounded rotor connects to the power grid through a backto-back converter. Consequently, the output currents are the combination of stator and grid-side converter currents, as shown in Fig.9.

Current control of DFIG under severe short-circuit faults is shown in Fig.10. Objective of the current control is to regulate

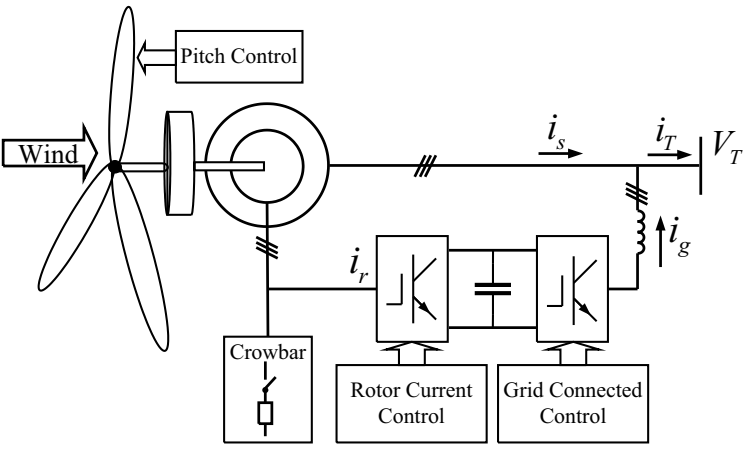

Fig. 9. Structure of DFIG.

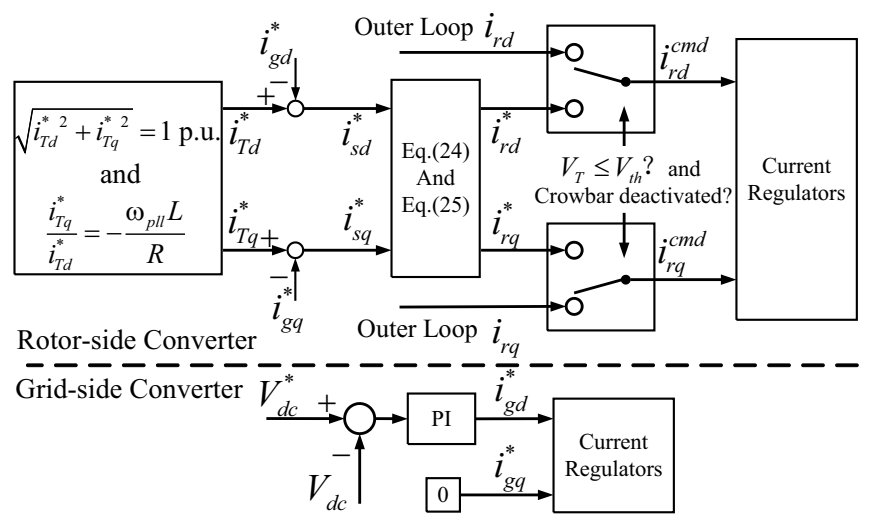

Fig. 10. Current control block diagram of DFIG.

the d-axis and q-axis components of $i_{T}$ in compliance with the adaptive current injecting strategy. DC voltage of the back-toback converter is supposed to be controlled as constant by the grid-side converter. Thus, we can obtain

$$
\begin{aligned}
& i_{s d}^{*}=i_{T d}^{*}-i_{g d}^{*} \\
& i_{s q}^{*}=i_{T q}^{*}-i_{g q}^{*}
\end{aligned}
$$

where $i_{T d}^{*}$ and $i_{T q}^{*}$ are the target currents deduced by the adaptive current injecting strategy. $i_{g d}^{*}$ is the grid-side converter $\mathrm{d}$-axis current command generated by the dc voltage control loop. The reactive current $i_{g q}^{*}$ of grid-side converter is often set to zero. Thus, $i_{s d}^{*}$ and $i_{s q}^{*}$ are the desired stator currents. However, only rotor currents of the DFIG is controllable. The relationships between rotor currents and stator currents of DFIG are shown as follow.

$$
\begin{aligned}
& i_{r d}^{*}=-\frac{L_{s}}{L_{m}} i_{s d}^{*} \\
& i_{r q}^{*}=\frac{1}{L_{m}} \Psi_{s}-\frac{L_{s}}{L_{m}} i_{s q}^{*}
\end{aligned}
$$

where $L_{s}=L_{l s}+L_{m} . L_{l s}$ and $L_{m}$ are stator leak inductance and mutual inductance, respectively. Stator flux $\Psi_{s}$ could be obtained via flux observer, which has been widely studied in motor drive area. (25) shows a simple flux observer.

$$
\Psi_{s}=\int\left(u_{s}+i_{s} R_{s}\right) d t
$$

As a result, the desired currents of wind farm are converted to the rotor current commands of DFIG, which could be regulated by the rotor-side converter. 
It should be mentioned that crowbar is utilized in DFIG to protect over-current when faults occur. When the faults occur, the crowbar is connected and the rotor-side converter is blocked. As a result, the DFIG works as an asynchronous machine and hence the currents of DFIG is uncontrollable during this stage. After several tens of milliseconds when crowbar is switched off and rotor-side converter is reconnected, output currents are again controllable and should be regulated according to Fig.10.

\section{Simulation VERIFICATION}

Fig.1 is established using MATLAB/Simulink. Electromagnetical dynamic are considered to validate the effectiveness of the analysis and the proposed control methods. The converters are represented by average-value models. The parameters of test system are given in APPENDIX.

\section{A. Steady State Working Point Validation}

From (6) and the analysis in Section III-A, let $\hat{v}_{q}=0$, then we can obtain two zero-crossing points. According to the small signal analysis in Section III-A, the $\delta_{0}-\phi_{0}<\frac{\pi}{2}$ zero-crossing point is the stable one. Consequently, we can get the steady state $\delta_{0}$ under prefault and fault condition, respectively.

$$
\begin{aligned}
\delta_{0} & =\phi_{0}+\arcsin \frac{a\left(\omega_{p l l}\right)}{b} \\
& =\angle K_{v}\left(\omega_{g}\right)+\arcsin \frac{\left|Z_{i}\left(\omega_{g}\right) I_{c}^{p}\right| \sin \left(\phi_{c}+\angle Z_{i}\left(\omega_{g}\right)\right)}{\left|K_{v}\left(\omega_{g}\right) E\right|}
\end{aligned}
$$

Particularly, when adaptive current injecting control law is applied, term $a\left(\omega_{p l l}\right)=0$. Hence

$$
\delta_{0}=\phi_{0}=\angle K_{v}\left(\omega_{g}\right)
$$

Fig.11(e) shows the $\delta$ curve. The steady state working points are also illustrated in Fig.11(e). Details of Fig.11 would be introduced in next part. With the parameters shown in APPENDIX, $\delta_{0}^{p r}$ and $\delta_{0}^{f a}$ are 0.4499rad and 4.4619rad, respectively. The simulation results match the calculated results exactly.

\section{B. Effectiveness of Adaptive Current Injecting Strategy}

This part illustrates feasibility of the adaptive current injecting strategy under a severe short-circuit fault. The shortcircuit fault is simulated by connecting a small resistance $R_{f}$ in parallel. PMSG and DFIG based wind farm are examined, respectively. The control strategies of PMSG and DFIG wind farm have been introduced in Section-V. PLL parameters in these two cases are set to $K_{p}=200$ and $K_{i}=50$. Note that the scale of $\hat{v}_{q}$ under fault state is much smaller compared to normal state. Hence, to ensure a similar bandwidth of PLL, here defines several voltage intervals. According to these voltage intervals, scheduled gain is applied to multiply $\hat{v}_{q}$. In the following simulation, voltage drops to lower than 0.1p.u. and the gain is 10 during the fault.
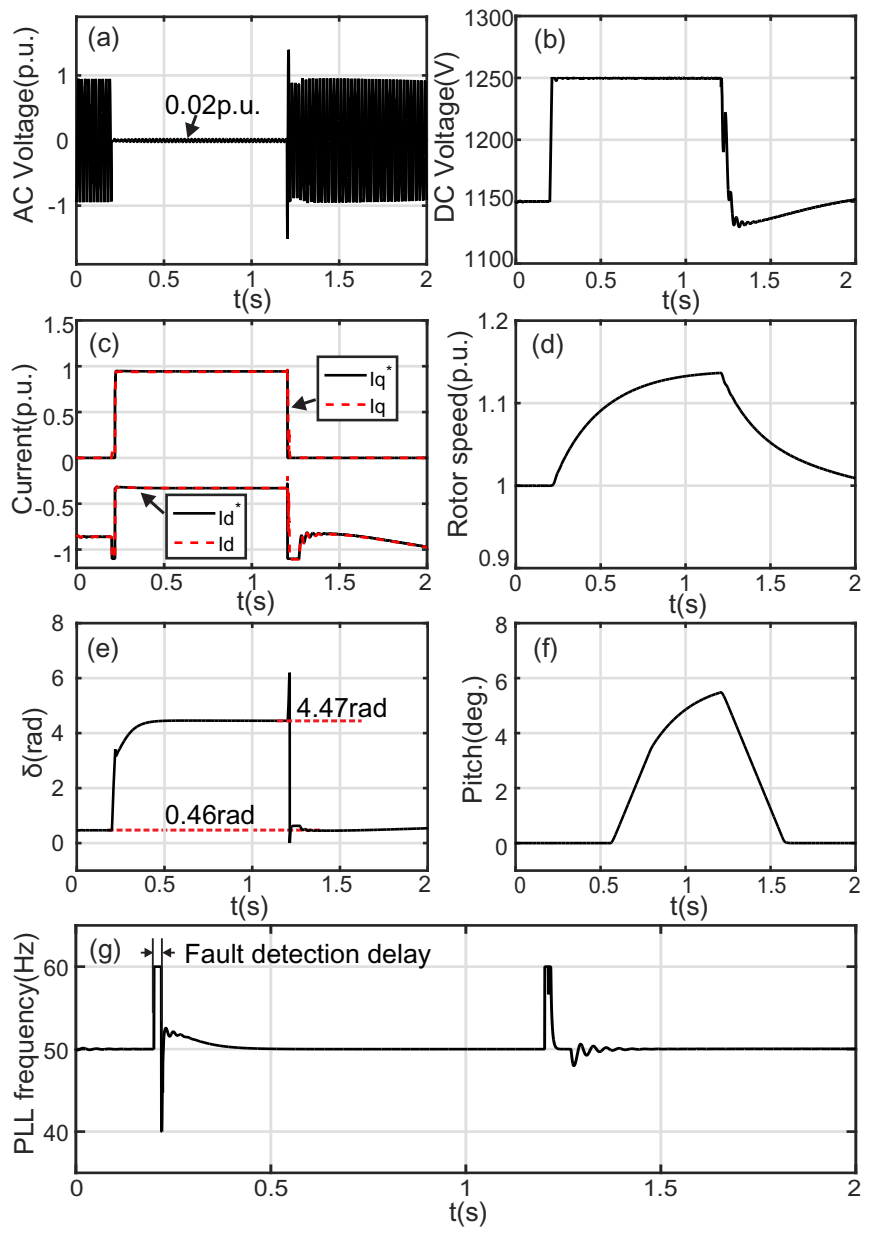

Fig. 11. Simulation results of PMSG.

1) PMSG: The short-circuit fault occurs in $t=0.2 \mathrm{~s}$. AC voltage drops to 0.02p.u, as shown in Fig.11(a). The generatorside converter controls electrical torque. At the same time, the dc chopper is activated in order to keep the dc bus below limitation, as shown in Fig.11(b). The grid-side converter could be treated as a current source injecting reactive and active currents in according to (16), as shown in Fig.11(c). In this case, electrical torque of the PMSG, controlled by the generator-side converter, is reduced when the fault occurs. Consequently, rotor speed will accelerate, activating pitch control, as shown in Fig.11(d) and Fig.11(f). By doing this, the input mechanical power is reduced and the burden on the dc chopper is then reduced. Fig.11(g) shows PLL frequency, which could converge to system frequency after transient process, validating the effectiveness of the adaptive current injecting strategy.

2) DFIG: This part validates the effectiveness of the proposed control strategy in DFIG wind farm. Two cases are established to show different characteristics under supersynchronous and subsynchronous conditions. Simulation results are shown as Fig.12 and Fig.13. In supersynchronous case, pitch control is activated to keep rotor speed below limitation. Desired currents obtained by adaptive current injecting strategy are the combination of stator currents and grid-side converter currents. Fig.12(d) and Fig.13(d) show that the rotor 

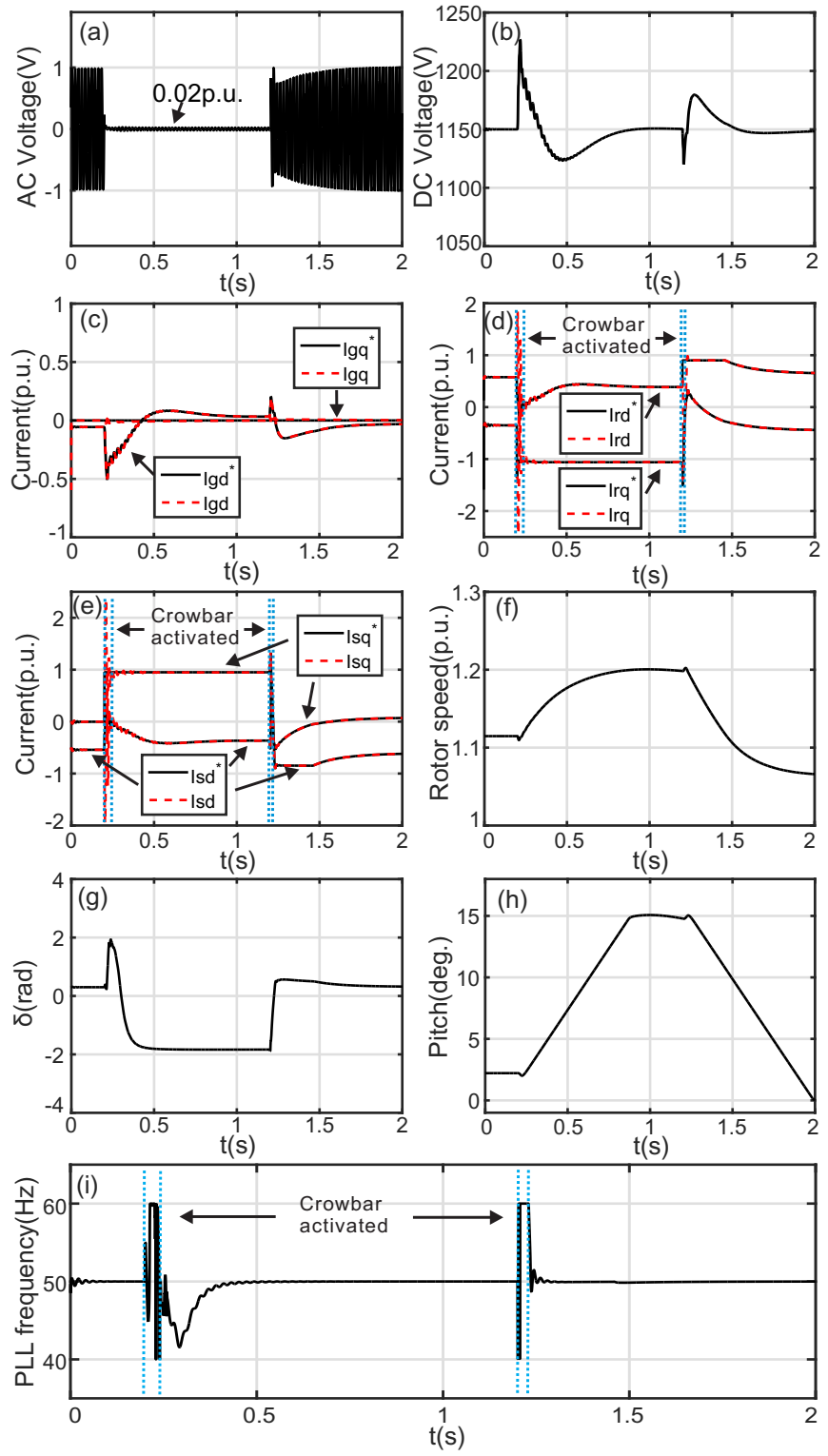

Fig. 12. Simulation results of DFIG under hypersynchronous condition.

currents could track the current commands exactly during the fault, hence, in Fig.12(e) and Fig.13(e) the stator currents could be regulated to desired value, as well as the grid-side converter currents, as shown in Fig.12(c) and Fig.13(c). As a result, the output wind farm currents could track the adaptive current injecting law, keeping wind farm synchronous with power grid during the severe short-circuit fault, as shown in Fig.12(i) and Fig.13(i). It should be mentioned that when the crowbar is activated, there is a drastic transient process. In both cases examined here, the transient process is less than $30 \mathrm{~ms}$. After the crowbar is deactivated, the rotor-side converter reconnects and the value of PLL integrator is set to zero manually.

\section{Robustness of Adaptive Current Injecting Strategy}

The proposed current injecting method need to know parameters $R_{\text {line }}$ and $L_{\text {line }}$ of the transmission line. However, weath-
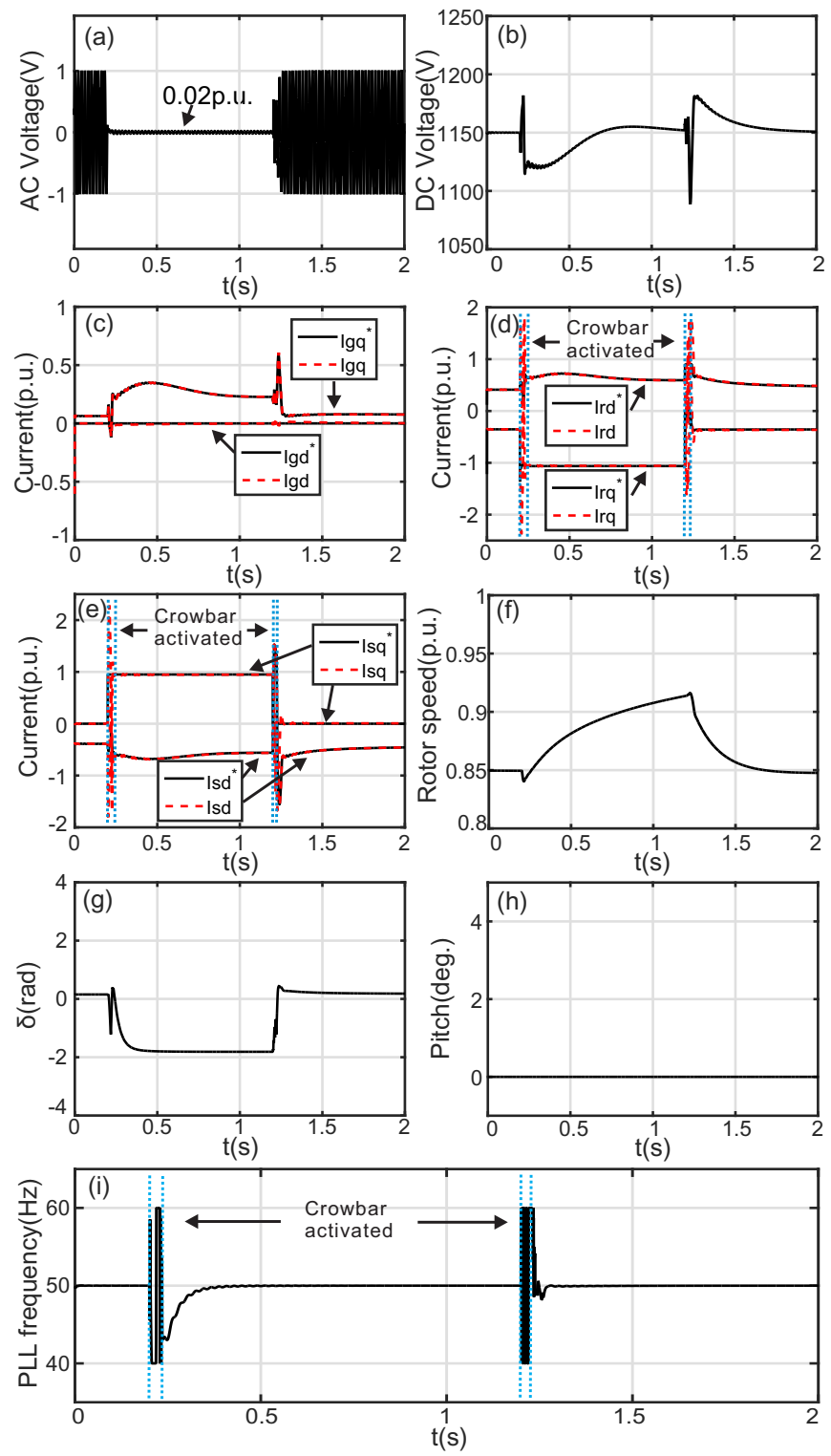

Fig. 13. Simulation results of DFIG under subsynchronous condition.

er, temperature, humidity and aging could lead to variation of the line parameters. Although the line parameters could be identified by measuring, precise parameters of the transmission line could not be obtained in practice. Hence, robustness of the proposed method is examined here to see whether the method can work with parameters mismatch caused by measuring error or other reasons.

Case studied here is the same as Fig.12. Parameters used in current injecting method is the normal value $R_{\text {line }}^{r}$ and $L_{\text {line }}^{r}$ shown in Table.I. The line parameters $R_{\text {line }}$ and $L_{\text {line }}$ are changed to examine the robustness, as shown in Fig.14. We can obtain that if the line parameters $R_{\text {line }}$ and $L_{\text {line }}$ locate in the blue line, $L / R$ ratio keeps unchanged and hence stability will not be affected. However, if $R_{\text {line }}$ increases(decreases) and $L_{\text {line }}$ decreases(increases), system is possible to get into the unstable region where PLL frequency can no longer converge to system frequency. In this case, the system is stable until variation of the parameters is up to $21 \%$, as the arrow in 


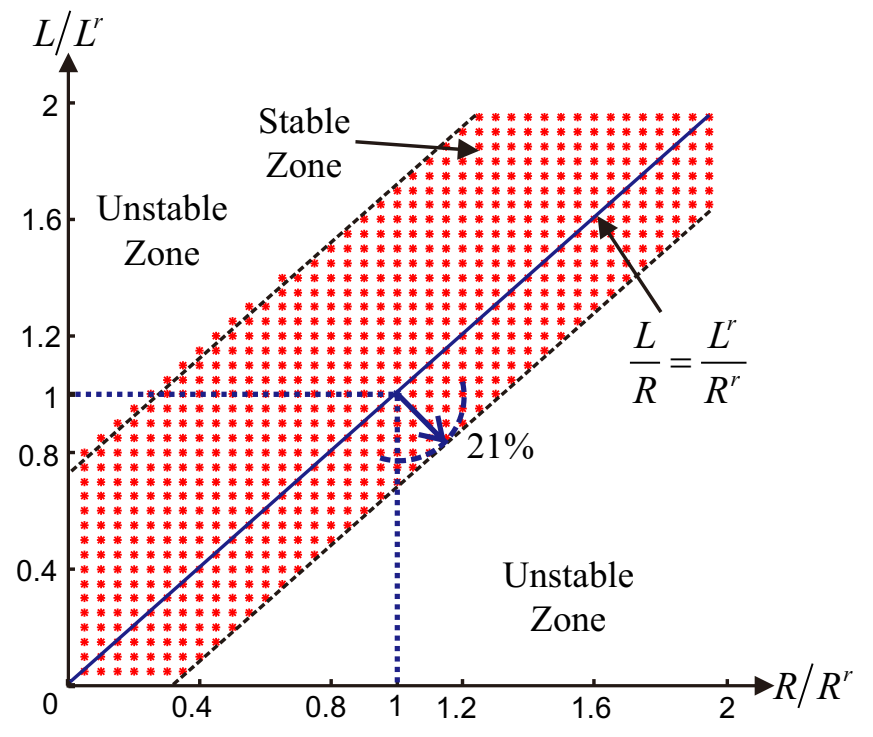

Fig. 14. Examination on robustness of the proposed method.

Fig.14. The simulation result shows that the proposed method can work without knowing precise line parameters.

\section{CONCLUSION}

Severe short-circuit faults make connection between wind farm and power grid weak, even though the connection of the prefault system is strong. Hence synchronization issues arise during the faults. In this paper, the dynamic process of grid-synchronization is described as motion of autonomous second-order nonlinear system with specific initial states. To ensure synchronization stability, special requirements for wind farm should be met. Moreover, the adaptive current injecting method is proposed, which can satisfy all the requirements. Simulations on both PMSG and DFIG based wind farm are then illustrated. Impact of detection delay and protection is considered in the simulations to better reflect a real-world scenario. Simulation results show that output currents of the wind farm could exactly track current commands generated by the adaptive current injecting method during the severe grid fault. Hence synchronization stability could be ensured and the method is verified. Robustness examination shows that the proposed method can work under a certain amount of parameters mismatch, which is $21 \%$ in the case examined in this paper.

Note that this paper uses an aggregated model to study the grid-synchronization issue. Actually, in wind farm, feeders and collection cables will lead to voltage difference between wind turbines, which is neglected in this paper. Hence, further work in detailed wind farm considering individual wind turbines and their connections should be elaborated. This would be the future work on the grid-synchronization issue.

\section{APPENDIX}

\section{A. Parameters of wind farm}

Rated frequency $f_{n}: 50 \mathrm{~Hz}$

Power base $S_{\text {base }}$ : 100MVA
Voltage base $V_{\text {base }}: 575 \mathrm{~V}$

Rated power of wind farm $P_{n}: 100 \mathrm{MW}$

Rated voltage of $E: 575 \mathrm{~V}$

Short-circuit ratio(SCR): 2

Resistance of transmission line $R_{\text {line }}: 0.0369$ p.u.

Inductance of transmission line $L_{\text {line }}: 0.1056 \mathrm{p} . \mathrm{u}$.

Resistance of grid impedance $R_{g}: 0.0546 \mathrm{p} . \mathrm{u}$.

Inductance of grid impedance $L_{g}: 0.382$ p.u.

Fault resistance $R_{f}$ : 0.008p.u.

\section{B. Parameters of PMSG}

Resistance $R_{s}$ : 0.006p.u.

Inductance in d-axis $L_{d}: 1.305$ p.u.

Inductance in q-axis $L_{q}: 0.296 \mathrm{p} . \mathrm{u}$.

Grid coupling inductance $L_{c}: 0.3$ p.u.

Generator inertia constant: $0.62 \mathrm{~s}$

Turbine inertia constant:4.32s

GSC voltage loop: $K_{p}=15, K_{i}=27.5$

GSC reactive power loop: $0.05, K_{i}=20$

GSC current loop: $K_{p}=1.2, K_{i}=1.25$

RSC speed loop: $K_{p}=5, K_{i}=1$

RSC current loop: $K_{p}=0.025, K_{i}=100$

\section{Parameters of DFIG}

Stator resistance $R_{s}: 0.023$ p.u.

Stator inductance $L_{s}: 0.18$ p.u.

Rotor resistance $R_{r}: 0.016$ p.u.

Rotor inductance $L_{r}: 0.16$ p.u.

Mutual inductance $L_{m}: 2.9$ p.u.

Generator inertia constant: $0.685 \mathrm{~s}$

Turbine inertia constant:4.32s

Grid coupling inductance $L_{c}$ : 0.3p.u.

GSC voltage loop: $K_{p}=6.4, K_{i}=32$

GSC reactive power loop: $0.05, K_{i}=20$

GSC current loop: $K_{p}=0.8, K_{i}=2.5$

RSC speed loop: $K_{p}=3, K_{i}=0.06$

RSC current loop: $K_{p}=2.4, K_{i}=0.8$

\section{REFERENCES}

[1] T. Ackermann, Wind power in power systems. Chichester, UK: John Wiley, 2005.

[2] L. D. Zhang, L. Harnefors, and H. P. Nee, "Power-synchronization control of grid-connected voltage-source converters," IEEE Trans. Power Syst., vol. 25, no. 2, pp. 809-820, 2010.

[3] X. Z. Xi, H. Geng, and G. Yang, "Enhanced model of the doubly fed induction generator-based wind farm for small-signal stability studies of weak power system," IET Renew. Power Gen., vol. 8, no. 7, pp. 765-774, 2014.

[4] J. Z. Zhou, H. Ding, S. T. Fan, Y. Zhang, and A. M. Gole, "Impact of short-circuit ratio and phase-locked-loop parameters on the small-signal behavior of a vsc-hvdc converter," IEEE Trans. Power Del., vol. 29, no. 5, pp. 2287-2296, 2014.

[5] O. Goksu, R. Teodorescu, C. L. Bak, F. Iov, and P. C. Kjaer, "Instability of wind turbine converters during current injection to low voltage grid faults and pll frequency based stability solution," IEEE Trans. Power Syst., vol. 29, no. 4, pp. 1683-1691, 2014.

[6] L. D. Zhang, L. Harnefors, and H. P. Nee, "Interconnection of two very weak ac systems by vsc-hvdc links using power-synchronization control," IEEE Trans. Power Syst., vol. 26, no. 1, pp. 344-355, 2011.

[7] B. Wen, D. Dong, D. Boroyevich, R. Burgos, P. Mattavelli, and Z. Y. Shen, "Impedance-based analysis of grid-synchronization stability for three-phase paralleled converters," IEEE Trans. Power Electron., vol. 31 , no. 1, pp. 26-38, 2016. 
[8] D. Dong, B. Wen, D. Boroyevich, P. Mattavelli, and Y. S. Xue, "Analysis of phase-locked loop low-frequency stability in three-phase grid-connected power converters considering impedance interactions," IEEE Trans. Ind. Electron., vol. 62, no. 1, pp. 310-321, 2015.

[9] S. Xiao, G. Yang, H. L. Zhou, and H. Geng, "An lvrt control strategy based on flux linkage tracking for dfig-based wecs," IEEE Trans. Ind. Electron., vol. 60, no. 7, pp. 2820-2832, 2013.

[10] J. Morren and S. W. H. de Haan, "Ridethrough of wind turbines with doubly-fed induction generator during a voltage dip," IEEE Trans. Energy Convers., vol. 20, no. 2, pp. 435-441, 2005.

[11] A. Mullane, G. Lightbody, and R. Yacamini, "Wind-turbine fault ridethrough enhancement," IEEE Trans. Power Syst., vol. 20, no. 4, pp. 1929-1937, 2005.

[12] D. Jovcic, "Phase locked loop system for facts," IEEE Trans. Power Syst., vol. 18, no. 3, pp. 1116-1124, 2003.

[13] P. Rodriguez, J. Pou, J. Bergas, J. I. Candela, R. P. Burgos, and D. Boroyevich, "Decoupled double synchronous reference frame pll for power converters control," IEEE Trans. Power Electron., vol. 22, no. 2, pp. 584-592, 2007.

[14] S. Golestan, J. M. Guerrero, A. Vidal, A. G. Yepes, and J. DovalGandoy, "Pll with maf-based prefiltering stage: Small-signal modeling and performance enhancement," IEEE Trans. Power Electron., vol. 31, no. 6, pp. 4013-4019, 2016.

[15] H. Geng, J. B. Sun, S. Xiao, and G. Yang, "Modeling and implementation of an all digital phase-locked-loop for grid-voltage phase detection," IEEE Trans. Ind. Informat., vol. 9, no. 2, pp. 772-780, 2013.

[16] H. Xin, L. Huang, L. Zhang, Z. Wang, and J. Hu, "Synchronous instability mechanism of $\mathrm{p}$-f droop-controlled voltage source converter caused by current saturation," IEEE Trans. Power Syst., vol. PP, no. 99, pp. 1-2, 2016.

[17] D. Dong, B. Wen, P. Mattavelli, D. Boroyevich, and Y. Xue, "Gridsynchronization modeling and its stability analysis for multi-paralleled three-phase inverter systems," in Proc. APEC, 2013, pp. 439-446.

[18] B. Wen, D. Boroyevich, P. Mattavelli, Z. Shen, and R. Burgos, "Influence of phase-locked loop on input admittance of three-phase voltage-source converters," in Proc. APEC, 2013, pp. 897-904.

[19] D. Dong, J. Li, D. Boroyevich, P. Mattavelli, I. Cvetkovic, and Y. Xue, "Frequency behavior and its stability of grid-interface converter in distributed generation systems," in Proc. APEC, 2012, pp. 1887-1893.

[20] L. M. Fernandez, F. Jurado, and J. R. Saenz, "Aggregated dynamic model for wind farms with doubly fed induction generator wind turbines," Renewable Energy, vol. 33, no. 1, pp. 129-140, 2008.

[21] L. M. Fernandez, C. A. Garcia, J. R. Saenz, and F. Jurado, "Equivalent models of wind farms by using aggregated wind turbines and equivalent winds," Energy Conversion and Management, vol. 50, no. 3, pp. 691704, 2009.

[22] M. Mohseni, S. Islam, and M. A. S. Masoum, "Fault ride-through capability enhancement of doubly-fed induction wind generators," IET Renew. Power Gen., vol. 5, no. 5, pp. 368-376, 2011.

[23] Q. $\mathrm{Hu}, \mathrm{J}$. Hu, H. Yuan, and H. Tang, "Synchronizing stability of dfigbased wind turbines attached to weak ac grid," in Proc. ICEMS, 2014, pp. 2618-2624.

[24] H. K. Khalil and J. Grizzle, Nonlinear systems. New Jersey: Prentice hall, 1996.

[25] L. Harnefors and H. P. Nee, "A general algorithm for speed and position estimation of ac motors," IEEE Trans. Ind. Electron., vol. 47, no. 1, pp. 77-83, 2000.

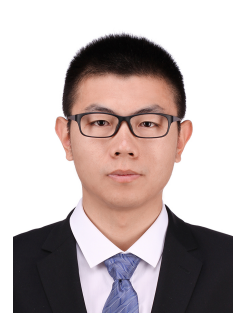

ShaoKang Ma (S'14) received the B.S. degree in College of Electrical and Information Engineering, Hunan University ,Changsha, China, in 2012 and the M.S. degree in Department of Automation, Tsinghua University, Beijing, China, in 2015. Since 2015, He is currently working toward the Ph.D. degree at Department of Automation, Tsinghua University.

His current research interests include distribution generation systems, renewable energy conversion systems, and digital control systems.

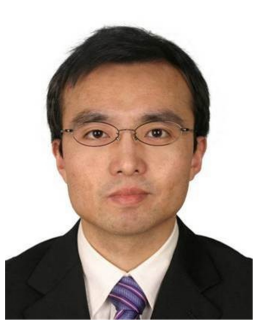

Hua Geng (S07-M10-SM14) received the B.S. degree in electrical engineering from Huazhong University of Science and Technology, Wuhan, China, in 2003 and the Ph.D. degree in control theory and application from Tsinghua University, Beijing, China, in 2008

From 2008 to 2010, he was a Postdoctoral Research Fellow with the Department of Electrical and Computer Engineering, Ryerson University, Toronto, ON, Canada. He joined Automation Department of Tsinghua University in June 2010 and now is an Associate Professor. His current research interests include advanced control on power electronics and renewable energy conversion systems. Dr. Geng serves as an editor of IEEE Trans. on Energy Conversion, an associate editor of IEEE Trans. on Industry Applications and an associate editor of Chinese Journal of Electrical Engineering.

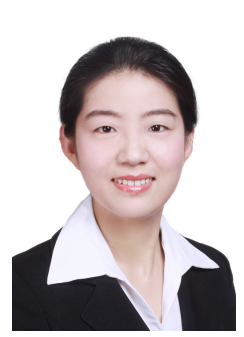

Lu Liu received the B.S. degree in School of $\mathrm{Au}-$ tomation Wuhan University of Technology, Wuhan, China, in 2014 and continued working toward the M.S. degree up to now.

Her current research interests include renewable energy conversion systems and Power electronic transformation and control technology.

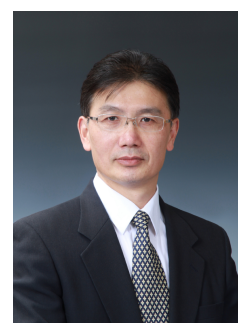

Geng Yang (M'01-SM'02) received the B.S. and M.S. degrees in electrical engineering from Xian University of Science and Technology, Xian, China, in 1982 and 1984, respectively, and the Ph.D. degree in electrical engineering from Sophia University, Japan, in 1992.

From 1985 to 1987, he was an Assistant with Xian University of Science and Technology. He was a Visiting Scientist with Fukui State University, Fukui, Japan, in 1987 and with Sophia University in 1988. From 1992 to 1994, he was a Senior Researcher with Kasuga ElectricalWorks Ltd., Japan. From 1995 to 1999, he was a Lecturer, Associate Professor, and Professor with Xian University of Science and Technology. Since 2000, he has been a Professor with the Department of Automation, Tsinghua University, Beijing, China. His current research interests include electrical drive and system, power electronic equipment, and control technology of wind and photovoltaic energy conversion systems.

Dr. Yang is a member of the Institute of Electrical Engineers of Japan and the Board Director of China Power Electric Institute. He is a member of China Electrotechnical Society (CES).

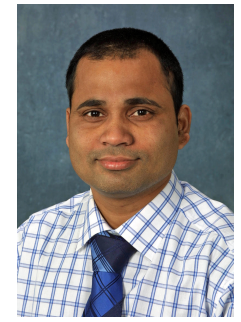

Bikash C. Pal received the B.Eng. degree from Jadavpur University, Calcutta, India, the M.Eng. degree from the Indian Institute of Science Bangalore, India, and the Ph.D. degree from Imperial College London, London, respectively, in 1990, 1992, and 1996, all in electrical engineering.

$\mathrm{He}$ is a Professor of Power Systems at Imperial College London. His research interest focuses on robust control of power systems, FACTS controllers, wide area monitoring and analysis, dynamic modeling, stability, and control of renewable generation, and distribution system state estimation. He is Editor-in-Chief of IEEE Trans. on Sustainable Energy and Fellow of IEEE for his contribution to power system stability and control. 